\title{
Diclofenac induced sudden sensorineural hearing loss
}

\section{Bhanukumar, Vineetha Bharathan Menon', Justin Kurian', Madhan Ramesh ${ }^{1}$ \\ Departments of General Medicine and ${ }^{1} \mathrm{Clinical}$ Pharmacy, JSS Hospital and Medical College, JSS University, Mysore, Karnataka, India}

Address for the Correspondence:

Dr. M. Bhanukumar, 12213 Second Phase, Fourth Stage, Vijayanagara, Mysore - 570 017, Karnataka, India.

E-mail: drmbk1961@gmail.com

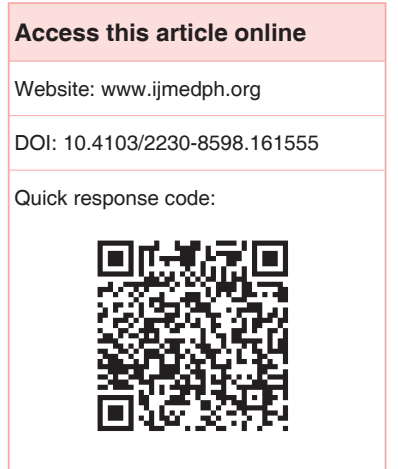

A few cases of mild to moderate, gradual and reversible sensorineural hearing loss (SNHL) with prolonged doses of nonsteroidal anti-inflammatory drugs has been reported. We present a case of sudden irreversible SNHL in a 60-year-old female after taking a single dose of diclofenac. The patient was a known case of diabetes mellitus and hypertension and was on regular treatment. We postulate that the patient's hearing loss was the result of diclofenac's ototoxic effects which may have been potentiated as a result of her long standing diabetes, hypertension and old age. Accordingly, we recommend the cautious use of diclofenac in patients with underlying diseases where diclofenac induced ototoxicity could potentially result in adverse otologic consequences.

Key words: Adverse drug reactions, diclofenac, nonsteroidal anti-inflammatory drugs, sensorineural hearing loss

\section{INTRODUCTION}

Nonsteroidal anti-inflammatory drugs (NSAIDs) are one of the most commonly used medications in everyday clinical practice. ${ }^{[1]}$ It is recommended for the long-term treatment of rheumatoid arthritis, osteoarthritis and ankylosing spondylitis and short-term treatment of acute musculoskeletal injury, postoperative pain and dysmenorrhea. ${ }^{[2]}$ Although they are reported to be safe in most cases, side effects do occur in some patients. Common side effects include gastric mucosal injury, renal impairment, allergic reactions and cardiovascular complications. In addition to these, high doses of NSAIDs are occasionally known to cause ototoxicity. ${ }^{[1]}$ Herein, we report a case demonstrating a rare and serious complication of NSAIDs, sudden sensorineural hearing loss (SSNHL).

\section{CASE REPORT}

The case report below was prepared in accordance with the principles in the Declaration of Helsinki. A 60-year-old female patient presented to the medicine unit with complaints of intermittent fever with severe myalgia since the past 5 days. Her past medical and medication history was collected. She was a documented case of hypertension and diabetes since 9 years and was on glimepiride, metformin, telmisartan and hydrochlorothiazide. She was initially injected diclofenac $75 \mathrm{mg}$ and her regular antihypertensives and antidiabetic medications were continued. Immediately within an hour after taking one dose of diclofenac, patient developed bilateral hearing loss, followed by tinnitus $24 \mathrm{~h}$ later. Patient had no history of any trauma, ear discharge or any symptoms relating to hearing difficulty in the past and no history of exposure to very loud noise or pesticides.

On examination, the patient was found to be afebrile, morbidly obese with body mass index of $43 \mathrm{~kg} / \mathrm{m}^{2}$. Her heart rate was 80 beats $/ \mathrm{min}$, regular and equally felt in all the peripheries. Her blood pressure was $140 / 90 \mathrm{mmHg}$ in right arm and supine position and had no variation in the left arm or on standing. Patient had no pallor, icterus, cyanosis, lymphadenopathy, edema and her systemic investigations were unremarkable. Fundus was normal. Her fasting glucose, postprandial blood glucose and glycated hemoglobin were $112 \mathrm{mg} / \mathrm{dL}, 186 \mathrm{mg} / \mathrm{dL}$ and 6.2, respectively, and within normal limits. Computerized tomography scan, magnetic resonance imaging of the brain and all other laboratory parameters were unexceptional. Patient's audiogram and tympanogram showed bilateral severe to profound hearing loss [Figure 1]. In her ear, nose and throat examination, Rinne's 
test was found to be negative and decreased. Absolute bone conduction test on both sides and Weber's test was centralized. Patient's brainstem auditory evoked potentials proved bilateral SNHL [Figure 2].

Based on the patient's history, current presentation, audiologic findings and associated comorbidities, diagnosis of diclofenac induced SSNHL with viral fever with type II diabetes mellitus with hypertension was considered. Diclofenac was immediately withdrawn and she was started on steroids, injection methyl prednisolone for 5 days at a dose of $500 \mathrm{mg} /$ day followed by oral prednisone for 3 weeks, but no satisfactory improvement was observed in her hearing status, even during the 3 months follow-up. Patient was then put on hearing aid trail and was advised cochlear implants.

Causality assessment, as assessed by using both Naranjo's criteria and WHO probability scale, revealed that the SSNHL was probably due to diclofenac. Severity of the reaction was evaluated using modified Hartwig and Siegel scale and was categorized as severe (level 6 reaction).

\section{DISCUSSION}

SSNHL is a sudden hearing impairment of at least $30 \mathrm{~dB}$ across three contiguous frequencies occurring within 3 days. ${ }^{[3]}$ Risk factors associated with SNHL are multifactorial and mainly encompass family or genetic predisposition, social and environmental factors, chronic medical conditions and use of medications. ${ }^{[4]}$

NSAIDs are found to be cochleotoxic. They inhibit enzyme cyclooxygenase and decrease prostaglandin activity, potentially reducing cochlear blood flow. High doses, regular use, and prolonged duration of therapy with NSAIDs have been reported to be ototoxic in animal studies and in human case reports. ${ }^{[5]}$ Usually, the hearing loss is reversed once the drug therapy is terminated. ${ }^{[6]}$ While there are very few reports of diclofenac induced mild to moderate, gradual and reversible SNHL, ${ }^{[7,8]}$ our patient presented with severe, irreversible SSNHL after a single normal dose of diclofenac. Though

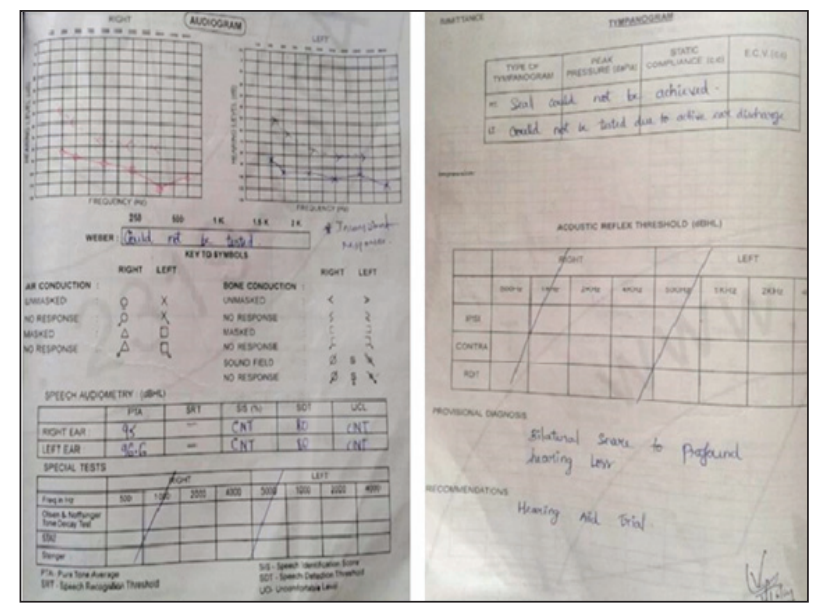

Figure 1: Patient's audiogram and tympanogram showing bilateral severe to profound hearing loss no evidence is available from the previous literature, each and every individual is liable to rare adverse reaction due to inter-individual variability. Another likely explanation for diclofenac ototoxicity is the altered pharmacokinetics due genetic factors. While standard doses of drugs are based on the pharmacokinetic data obtained from a pool of randomly selected healthy individuals, inherent differences between individuals may result in variations in drug metabolism. CYP2C9 contributes to the metabolism of NSAIDs like diclofenac. Impaired CYP2C9 metabolism may result in its increased plasma concentration leading to greater risk of adverse effects. CYP2C8 genotype also contributes to a small extent in NSAIDs metabolism. The two missense mutations, CYP2C $8 * 3$ and CYP2C $8 * 4$ yield enzymes with decreased activity. Although plausible, this hypothesis has not been adequately tested. ${ }^{[9]}$

While diclofenac is found to be associated with hearing loss, other insults to the cochlea from diabetes mellitus, hypertension and aging may also contribute to the decline in hearing experienced by the patient. Presbycusis or age-related hearing loss, which occurs due to degenerative changes in the cochlear hair cells and neurons in the cochlear nerve and its central auditory connections, ${ }^{[10]}$ is bilaterally symmetrical, usually insidious in onset, gradually progressing and starts from middle age and continues into older years. ${ }^{[4,10]}$ However, our patient had no history of hearing loss previously and the current presentation was a sudden onset. Our patient could have been at increased risk of diclofenac induced ototoxicity because of various old age related factors that contribute to drug accumulation in the body, increased sensitivity to adverse drug reactions and altered metabolism. ${ }^{[11]}$ Age-related changes in the patient's bile flow rate, bile composition, gastrointestinal and kidney functions ${ }^{[12]}$ may also alter the pharmacokinetic profile of diclofenac and increase the potential for adverse outcomes which could be another possible explanation for its ototoxicity.

Both long-term diabetes and hypertension have also been independently demonstrated to cause SNHL. In patients with diabetes, two possible mechanisms have been proposed,

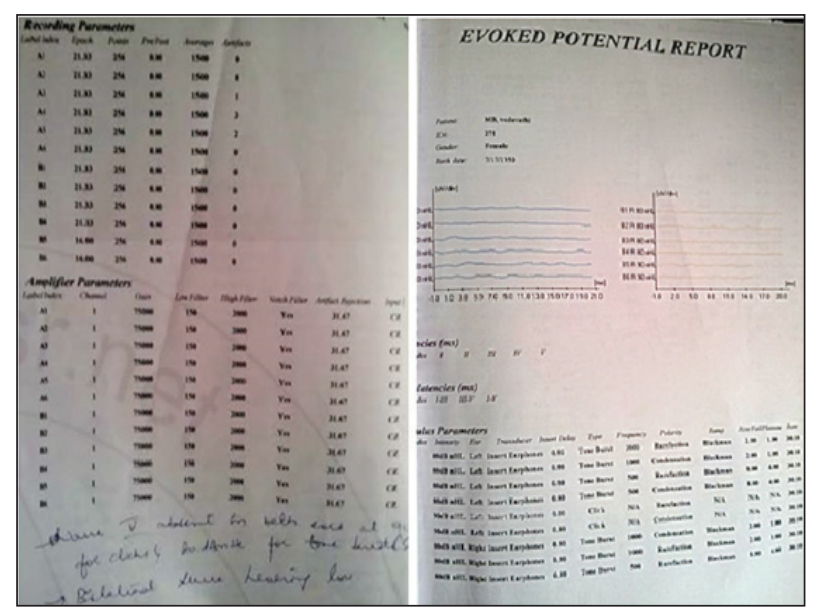

Figure 2: Patient's brainstem auditory evoked potentials showing bilateral sensorineural hearing loss 
microangiopathic lesions in the inner ear leading to cochlear hearing disorder or primary neuropathy of the cochlear nerve resulting in a retrocochlear hearing loss. ${ }^{[10]}$ Unlike our patient's sudden clinical presentation of SNHL, diabetes associated hearing loss is usually progressive, bilateral, sensorineural of gradual onset. ${ }^{[13]}$ Moreover, the patient's glycemic levels were controlled. Hypertension may also increase the risk of hearing loss due to changes in the cochlear microvasculature. ${ }^{[5]}$ High pressure in the vascular system can cause inner ear hemorrhage which may result in progressive or sudden hearing loss. ${ }^{[14]}$ However, patients computerized tomography scan and magnetic resonance imaging were normal. Thus, diabetes and hypertension associated hearing loss can be ruled out. Viral infections may also be considered as one of the major causes for hearing loss. Agents known to cause hearing loss include mumps, measles, rubella, Epstein Barr virus, herpes simplex, varicella zoster and cytomegalovirus. ${ }^{[15,16]}$ While our patient had a febrile illness, there were no specific physical signs and symptoms associated with these infections neither any symptoms of upper airway infections nor intracranial infections. Viral infections like measles, mumps, rubella and Epstein Barr virus do not occur in the elderly age group and cytomegalovirus occur in immunocompromised patients. Varicella zoster and herpes simplex can affect any age group, but there were no signs and symptoms of these infections in the patient. Moreover, the clinical presentation of SNHL in our patient is different from SNHL caused by viral infections which is usually characterized by vertigo, nausea, vomiting, difficulty in vision and impaired concentration. ${ }^{[17]}$ There are also no reports of SNHL caused by glimepiride, metformin, telmisartan and hydrochlorothiazide. Thus, based on the pharmacokinetics of diclofenac, the time temporal relationship for development of the event and clinical presentations, we strongly content that this clinical event of deafness was due to diclofenac.

SSNHL being an otologic emergency was treated with corticosteroids. It is considered as the gold standard of treatment. However, many studies fail to identify the value of steroids in the treatment of SNHL. ${ }^{[3]}$ In our patient, no response to steroids was observed. Alternative means of treating irreversible SNHL are hearing aids, cochlear implants, assistive listening devices and other aural rehabilitation methods. ${ }^{[4]}$ Hearing aids are usually the initial mode of treatment. ${ }^{[10]}$ Since the patient had severe to profound hearing loss as per the findings in audiogram and tympanogram, cochlear implants were advised.

\section{CONCLUSION}

Here we report a case of an otherwise healthy patient who experienced permanent SSNHL after a single dose of diclofenac. The time temporal relationship between prescribing diclofenac and development of hearing loss for the patient as along with the bilateral involvement, suggests diclofenac induced SSNHL rather than other causes. Consequently, physicians should be made aware of the potential for normal dose of diclofenac to cause irreversible SSNHL as a serious adverse drug reaction and should be cautious while prescribing it in patients with increased risk for ototoxicity, particularly diabetes, hypertension and advanced age.

\section{REFERENCES}

1. Hoshino T, Tabuchi K, Hara A. Effects of NSAIDs on the inner ear: Possible involvement in cochlear protection. Pharmaceuticals 2010;3:1286-95.

2. Kirchheiner J, Meineke I, Steinbach N, Meisel C, Roots I, Brockmöller J. Pharmacokinetics of diclofenac and inhibition of cyclooxygenases 1 and 2: No relationship to the CYP2C9 genetic polymorphism in humans. Br J Clin Pharmacol 2003;55:51-61.

3. Conlin AE, Parnes LS. Treatment of sudden sensorineural hearing loss: I. A systematic review. Arch Otolaryngol Head Neck Surg 2007;133:573-81.

4. Sogebi OA. Assessment of the risk factors for hearing loss in adult Nigerian population. Niger Med J 2013;54:244-9.

5. Curhan SG, Eavey R, Shargorodsky J, Curhan GC. Analgesic use and the risk of hearing loss in men. Am J Med 2010;123:231-7.

6. Brummett RE. Drug-induced ototoxicity. Drugs 1980;19:412-28.

7. Jahan S, Milky MF. Prevalence of analgesic induced deafness. Banglad J Neurosci 2011;27:74-7.

8. Kolodny AL. Two double blind trials of diclofenac sodium with aspirin and with naproxen in the treatment of patients with rheumatoid arthritis. J Rheumatol 1988;15:1205-11.

9. Janicki PK. Pharmacogenomics of pain management. In: Deer TR, Leong MS, Buvanendran A, Gordin V, Kim PS, Panchal SJ, et al., editors. Treatment of chronic pain by interventional approaches: The American academy of pain medicine textbook on patient management. $1^{\text {st }} \mathrm{ed}$. New York: Springer; c2015. p. 23-33.

10. Sogebi OA, Olusoga-Peters OO, Oluwapelumi O. Clinical and audiometric features of presbycusis in Nigerians. Afr Health Sci 2013; 13:886-92.

11. Coggins MD. Medication-related ototoxicity. Today's Geriatr Med 2014;7:p6. Available from: http://www.todaysgeriatricmedicine.com/ archive/052714p6.shtml. [Last accessed on 2015 Jan 04].

12. McLean AJ, Le Couteur DG. Aging biology and geriatric clinical pharmacology. Pharmacol Rev 2004;56:163-84.

13. Rajendran S, Anandhalakshmi, Mythili B, Rao V. Evaluation of the incidence of sensorineural hearing loss in patients with type 2 diabetes mellitus. Int J Biol Med Res 2011;2:982-7.

14. De Moraes Marchiori LL, de Almeida Rego Filho E, Matsuo T. Hypertension as a factor associated with hearing loss. Braz J Otorhinolaryngol 2006;72:533-40.

15. Lazarini PR, Camargo AC. Idiopathic sudden sensorineural hearing loss: Etiopathogenic aspects. Braz J Otorhinolaryngol 2006;72:554-61.

16. Merchant SN, Durand ML, Adams JC. Sudden deafness: Is it viral? ORL J Otorhinolaryngol Relat Spec 2008;70:52-60.

17. Shupert CL. Infections of the inner ear. Vestibular neuritis and labyrinthitis. Vestibular Disorders Association; c2013. p. 6. Available from: http://www. vestibular.org/sites/default/files/page_files/Documents/Vestibular $\% 20$ Neutitis\%20\%26\%20 Labyrinthitis.pdf. [Last accessed on 2015 Apr 14].

How to cite this article: Bhanukumar M, Menon VB, Kurian J, Ramesh M. Diclofenac induced sudden sensorineural hearing loss. Int J Med Public Health 2015;5:250-2.

Source of Support: Nil, Conflicts of Interest: None declared. 